\title{
A case with fistula formation between a perinephric retroperitoneal abscess, a ureter and a descending colon: Successful outcome after conservative management
}

\author{
Yoichi Iwamoto, MD; Masafumi Kato, MD \\ Department of Urology, Mie Central Medical Center, Mie, Japan
}

ite as: Can Urol Assoc J 2014;8(9-10):e644-6. http://dx.doi.org/10.5489/cuaj.1789 Published online September 9, 2014.

\section{Abstract}

A 72-year-old man diagnosed with hydronephrosis and perinephric abscess because of an impacted urinary stone in the left ureteropelvic junction was admitted to hospital with high-grade fever. The insertion of a percutaneous drain improved his general condition. Then, imaging from the percutaneous drainage tube revealed a fistula formation between the cavity of the perinephric retroperitoneal abscess, the descending colon, and the left renal pelvis. The patient received conservative management, including insertion of a percutaneous drain, discontinuation of oral intake and antimicrobial chemotherapy. Three days after the percutaneous drainage and discontinuation of oral intake, imaging revealed no apparent fistula. Fistula formation between a perinephric abscess and the descending colon is very rare; this is the first case of a long-term favourable outcome by conservative management.

\section{Introduction}

Retroperitoneal abscesses are occasionally encountered in hospital-based care. However, fistula formation between a retroperitoneal abscess, a ureter and a colon is rare. In such cases, surgery is usually curative. We report a case in which a fistula developed between a retroperitoneal abscess, a ureter and a descending colon; treatment was curative with conservative management.

\section{Case report}

A 72-year-old man with continuous high-grade fever and weight loss was transferred to our hospital. He was diagnosed with minor hydronephrosis and a perinephric abscess due to an impacted urinary stone with a diameter of $17 \mathrm{~mm}$ in the left uretero-pelvic junction (Fig. 1, part A). At admis- sion, although the patient had high fever, other vital signs were stable. An increasing inflammatory reaction with a white blood cell count of $9700 / \mu \mathrm{L}$ and a C-reactive protein value of $18.1 \mathrm{mg} / \mathrm{dL}$ were found by blood count and chemistry evaluation, respectively. Blood cultures and urine culture were both positive for Escherichia coli. The left percutaneous abscess drainage was constructed immediately after admission for the percutaneous drainage; $140 \mathrm{~mL}$ of light-white turbid fluid was obtained. The fluid culture was also positive for Escherichia coli. Contrast radiography of the abscess via the percutaneous drainage tube revealed a fistula into the descending colon and the left ureter (uretero-colic fistula) (Fig. 1, part C), while a barium enema of the colon revealed no apparent communication to the abscess (Fig. 1, part D). Soon thereafter, he became afebrile and his general condition improved.

Conservative treatment was chosen because the fistula tract was suspected to be imperceptible and the patient's performance status was poor: Eastern Cooperative Oncology Group (ECOG) Performance Status (PS) grade 4. Immediately after percutaneous drainage of the perinephric abscess, we began conservative treatment, including no oral intake, and antimicrobial chemotherapy with meropenem against potential disseminated bacterial infection. Three days after percutaneous drainage of the perinephric abscess, discharge was hardly observed and the patient became afebrile. Contrast radiography revealed a percutaneous drainage tube and no apparent communication between the abscess and fistula. Thereafter, oral intake was resumed. One week after percutaneous drainage of the perinephric abscess, contrast radiography revealed that the abscess had shrunk, because of efficacious percutaneous drainage, and the fistula remained undetectable. The percutaneous drainage tube to the perinephric abscess was then clamped, and 1 week later contrast radiography also revealed that the abscess had further shrinking and the fistula remained undetectable, and we stopped the antibiotics. After that, the percutaneous drain- 


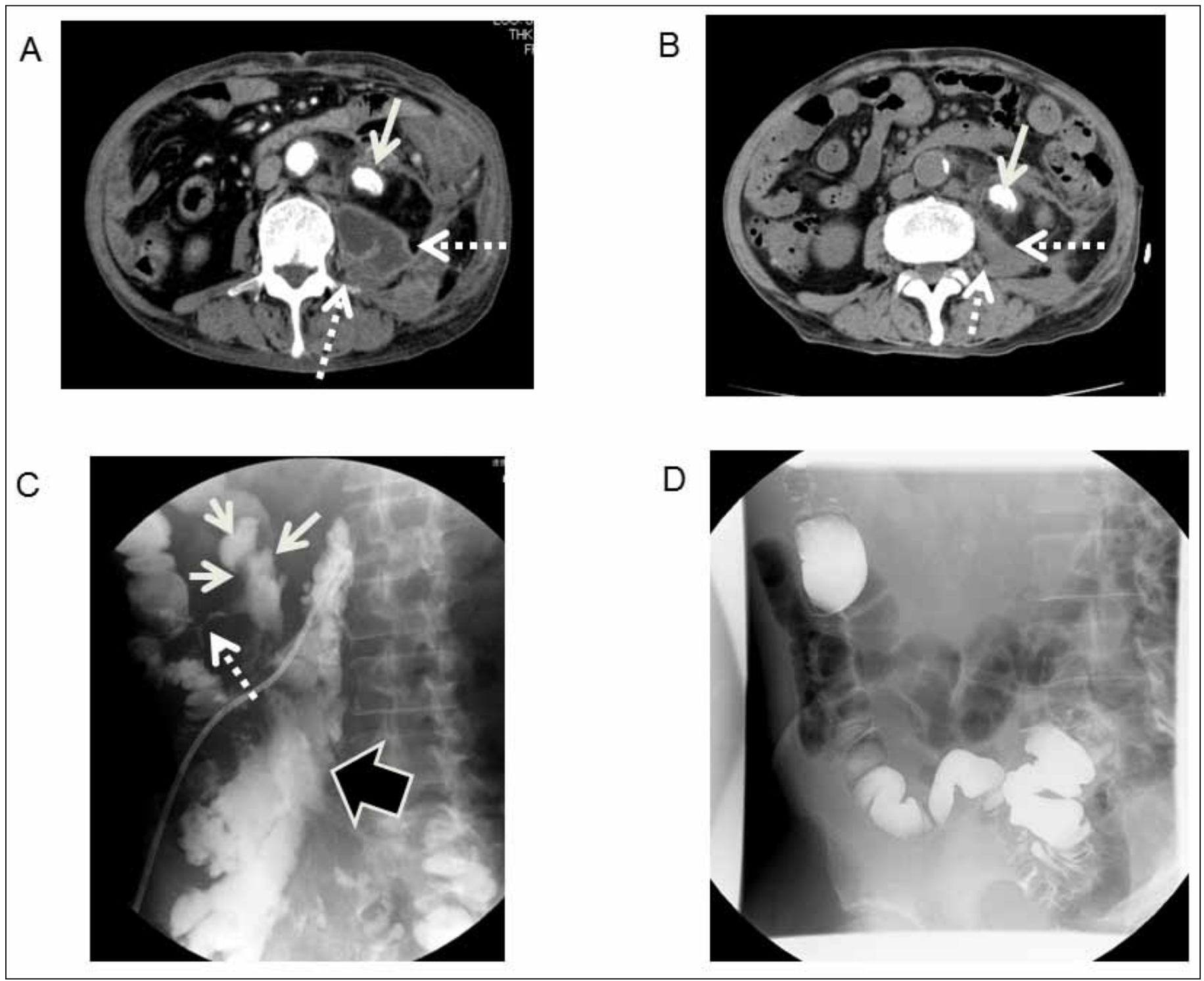

Fig. 1. (A) Contrast-enhanced computed tomography (CT) revealed the left ureter stone in the uretero-pelvic junction (solid arrow), and the perinephric abscess (dotted arrow). (B) Plain CT revealed abscess shrinkage for 6 months after treatment (dotted arrow). (C) Antegrade contrast radiography of an abscess (black arrow) revealed a fistula into the descending colon (dotted arrow), and the left hydronephrosis (solid arrow). (D) Barium enema of the colon revealed no apparent communication to the abscess.

age tube removed and the patient was discharged in good condition on post-drainage day 17 . At the 3 - and 6-month follow-up after percutaneous drainage, he did not suffer a relapse with computed tomography (Fig. 1, part B).

\section{Discussion}

Despite the close anatomical pathology between the upper urinary tract and colon, there have been few reports of fistula formation between them; only 10 cases since $1994 .^{1-5}$ The most common causes of spontaneous uretero-colic fistula are diverticulitis; causes for an obstructing ureter include ureteral calculi, tuberculosis, trauma, tumour and inflam- matory bowel disease. ${ }^{4}$ The suspected etiology in this case was that the impacted ureteral stone with urosepsis caused necrosis of the adjacent ureteral wall and the urosepsis inevitably perforated into an adjacent colon.

In cases of uretero-colic fistula, it has been recommended that nephroureterectomy with excision and closure of the fistula should be pursued if the affected kidney is non-functioning. ${ }^{2}$ If the kidney is functioning, and the contralateral kidney has a reduced functional capacity, removal of the fistulous tract and reanastomosis of the ureter should be considered. ${ }^{2}$ It has also been reported that adequate drainage and antibiotic therapy may lead to spontaneous closure of a uretero-colic fistula secondary to calcuous pyohydro- 
ureteronephrosis. ${ }^{4}$ Quantification of the degree of residual renal function within the affected kidney after relief of the obstruction is important to plan the definitive surgery. If there is poor renal function after relief of the ureteral obstruction, a nephroureterectomy, fistulectomy, segmental colonic resection and loop ileostomy ${ }^{5}$ are the definitive surgical treatment. If there is significant return of renal function or if there is a single kidney with some residual function, renal sparing surgery might be suitable. ${ }^{5}$ Choice of conservative or surgical management is also highly dependent on the general condition of the patient.

In our case, one reason why conservative management was successful is that the perinephric retroperitoneal space was limited and the dissemination was difficult. The fistula was small because the initial barium enema of the colon revealed no apparent communication. To our knowledge, there has been only one previous report describing the management of a uretero-colic fistula with conservative management (using ureteral stent); however, the patient died several days later from an unrelated cardiac event. ${ }^{4}$ This is the first case of long-term successful outcome after conservative management with percutaneous abscess drainage. This patient had neither nephroureterectomy nor ureter lithotripsy with poor surgical risk; however, he fortunately did not notice any symptoms because there was minor renal pelvis expansion by the disruption of the left kidney at follow-up 6 months after the percutaneous drainage.

\section{Conclusion}

Although we could not clearly distinguish the best treatment, conservative management with percutaneous drainage is a good alternative.

Competing interests: Dr. Iwamoto and Dr. Kato all declare no competing financial or personal interests.

This paper has been peer-reviewed.

\section{References}

1. Cirocco WC, Priolo SR, Golub RW. Spontaneous ureterocolic fistula: A rare complication of colonic diverticular disease. Am Surg 1994;60:832-5.

2. Maeda Y, Nakashima S, Misaki T. Ureterocolic fistula secondary to colonic diverticulitis. Int I Urol 1998;5:610-2. http://dx.doi.org/10.1111/j.1442-2042.1998.tb00422.x

3. Shiomi S, Kawabe J, Torii K, et al. Ureterocolic fistula detected on bone scintigraphy. Indian I Gastroenterol 2004;23:38-9. http://bir.biriournals.org/content/78/934/954.long

4. Lee WK, Chang SD, Roche CJ, et al. Spontaneous ureterocolic fistula secondary to calculous pyohydroureteronephrosis. Br J Radiol 2005;78:954-5. http://dx.doi.org/10.1259/bir/53711174

5. Dowling $C M$, Floyd MS, Power RE, et al. Ureterocolic fistula in the presence of a solitary kidney. BMJ Case Rep 2009;2009. http://www.ncbi.nlm.nih.gov/pmc/articles/PMC3030306/

Correspondence: Dr. Yoichi Iwamoto, Department of Uology, Mie Central Medical Center, Mie, Japan, 2185-5 Hisaimyoïncho, Tsu, Mie 514-1101, Japan; yoichi_iwamoto@yahoo.co.jp 